\title{
LE TRAVERSATE DELLE MI- GRAZIONI STORICHE ITALIANE TRA EVENTO E RACCONTO
}

The Crossing of Italian "historical" migration between lived experience and narration

\author{
Augusta \\ MOLINARI \\ Università di Genova \\ augusta.molinari@unige.it
}

\begin{abstract}
RESUMO
Il viaggio di emigrazione è un oggetto d'indagine storica sfuggente: è una storia di uomini, di navi, di interessi economici e politici. Per gli emigranti è soprattutto un momento di sospensione tra la vita che si sono lasciati alle spalle e quella nuova che troveranno nei paesi di destinazioni. Per gli intellettuali la traversata a fini di conoscenza (letteraria, scientifica, sociologica) è il luogo per eccellenza da cui osservare i flussi migratori e lasciarne una narrazione. La traversata dei migranti tra Ottocento e Novecento può diventare un osservatorio interessante per verificare l'intreccio, spesso drammatico, tra i progetti migratori dei ceti subalterni e le dinamiche di sfruttamento in atto nei loro confronto sia in Italia sia nelle Americhe. Le infermerie delle navi, soprattutto nei viaggi di ritorno dagli Stati Uniti, sono affollate di emigranti che subiscono provvedimenti di rimpatrio coatto perché malati di tubercolosi o perché considerati malati mentali. Per chi, spesso al momento dello sbarco, veniva "respinto" in Italia perché malato di mente non c'era solo il fallimento del progetto migratorio ma il rischio di concludere in manicomio la propria esistenza.
\end{abstract}

Parole chiave: Migrazioni storiche italiane; Viaggio transoceanico; malattie degli emigranti.

\begin{abstract}
The crossings of Italian "historical" migrations between lived experience and narration. The emigration journey is a shifty subject of historical investigation. It's a story of men and women, of ships, and of economic-political interests. To migrants, it is above all a moment of suspension between the life they have left behind and the new one they will find in their respective countries of destination. To intellectuals, who experience the crossing as a form of knowledge - literary, scientific, sociological the journey is the vantage point par excellence from which to observe migration flows and narrate them. The migrants' crossings between the Nineteenth and Twentieth centuries, may become an interesting observatory to verify the often dramatic interlacing between the projects of people belonging to the lower orders and the dynamics of exploitation of which they were the objects both in Italy and the Americas. The sickbays, especially on ships returning from the United States, were packed with emigrants forcibly repatriated because affected by either tuberculosis or mental diseases. For those who were rejected and sent back to Italy - oftentimes at disembarkation - on account of mental illness, this meant not only the failure of the migration project but also the risk of spending the rest of their lives in a lunatic asylum.
\end{abstract}

Keywords: Italian historical migrations; transAtlantic journey; the emigration journey as narration; psycho-physical check up of emigrants. 


\section{Il viaggio di emigrazione come racconto}

I viaggio di emigrazione resta a tutt'oggi, nonostante l'interesse che i fenomeni migratori hanno riscosso in ambito storiografico, uno degli aspetti meno studiati delle migrazioni storiche italiane (MOLINARI, 1988; 2005; 2009; 2014). Le ragioni di questa lacuna sono molte e, solo in parte, attribuibili alla scarsa attenzione dedicata dagli storici a questo argomento. E' la natura di "cammino" e non di "luogo" che fa del viaggio di emigrazione più un soggetto letterario che un oggetto di indagine storica (FRANZINA, 2003; MOLINARI, 2014). Questo avviene non tanto perché del viaggio di emigrazione esistano narrazioni di viaggiatori "illustri", da Charles Dickens, a Robert Louis. Stevenson, a Edmondo De Amicis, oltre una vasta letteratura popolare e di divulgazione scientifica (BLENGINO, 1990; MARTELLI, 1994). Quanto, piuttosto, per la valenza che assume il viaggio nell'ambito delle esperienze migratorie. Per l'emigrante, a differenza che per il letterato, il viaggio non è un "cammino" ma solo la parentesi di un "cammino". E' con l'arrivo nel paese di destinazione che il viaggio assume il carattere di una tappa del percorso migratorio.

Il ruolo di parentesi tra la formulazione di un progetto migratorio e la sua realizzazione fa del viaggio transoceanico un oggetto di indagine difficile da definire. E' un aspetto importante della storia del trasporto marittimo, della storia economica, della storia delle élite politiche, economiche, culturali. Ma è una storia di navi, più che di emigranti. Di interessi economici, più che di vite (TONIZZI, 2000; MOLINARI, 2001, p. 237-250). Non è certo casuale sia così arduo documentare quale fosse la vita degli emigranti nel corso delle traversate. Tra i fattori che contribuiscono a rendere il viaggio poco "visibile" in ambito storiografico, $c^{\prime}$ è il disinteresse dello stato e delle istituzioni per il destino dei migranti. Almeno fino ai primi anni del Novecento sono poche le tracce del viaggio transoceanico rimaste nelle carte degli archivi. ${ }^{1}$

E', però, soprattutto, il carattere di "non luogo" (AUGÉ, 1993) che il viaggio assume per gli emigranti a farne un'esperienza difficile da documentare. Nel corso delle traversate quelle degli emigranti sono "vite sospese": si sono lasciati alle spalle una vita e non sanno come sarà quella che troveranno nel paese di destinazione. L'esperienza del viaggio è l'attesa di un nuovo destino di vita che solo il raggiungimento della meta migratoria fa apparire praticabile. Ciò che accade a bordo delle navi è vissuto come accidentale rispetto alla realizzazione del progetto migratorio. Il viaggio per mare e la vita di bordo suscitano stupore, spesso paura (FRANZINA, 1992 ). E', però, la lunga e spesso estenuante attesa dell'arrivo, più che le vicende della traversata, a definire la condizione e il vissuto degli emigranti nel corso del viaggio. Ciò che succede a bordo può essere bello o brutto, a volte è anche qualcosa di terribile come la morte, ma viene vissuto come una vicenda episodica e marginale rispetto alla finalità del viaggio. E' solo quando, a volte accade, la meta non viene raggiunta, che il viaggio diventa un'esperienza a sé nel percorso migratorio. O perché ci si ammala e si muore a bordo, o per i tanti inconvenienti che si verificano sia nei porti di imbarco sia nel 
corso delle traversate (MORICOLA, 2000; MOLINARI, 2014). C'è chi non riesce a imbarcarsi perché non supera la visita medica all'imbarco, chi viene frodato dagli agenti di emigrazione e non trova la nave su cui imbarcarsi, chi è derubato nelle locande degli angiporti, chi sale a bordo di navi talmente vecchie e in cattive condizioni che non sono in grado di giungere a destinazione. E' l'eccezionalità che fa assumere al viaggio il carattere di un evento. Un'eccezionalità che, in genere, segna la fine di un percorso migratorio o diventa un evento luttuoso che la pregiudica (STELLA, 2004; CAMPANINI, 2010).

Non è un caso che le traversate dell'emigrazione italiana di massa siano stato raccontate prevalentemente da coloro che non ne erano protagonisti ma testimoni. Settori diversi di ceti intellettuali che, parafrasando il titolo di una delle più note narrazioni del viaggio, quelle di Robert Louis Stevenson, erano emigranti per diletto (MOCHI, 1987). Oppure, da coloro, sempre appartenenti ai ceti intellettuali, che diventavano emigranti per professione: medici di bordo, geografi, antropologi. A differenza di quanto avviene per l'emigrante, il viaggio, per chi lo compie come testimone, è il "luogo" per eccellenza dei fenomeni migratori. In queste narrazioni la nave appare come un osservatorio sociale dove si intrecciano sguardi diversi: la pietas di tipo filantropico, la curiosità antropologica verso mondi subalterni poco conosciuti, la denuncia per le condizioni di sfruttamento nei paesi di immigrazione, le diverse opinioni dei ceti dirigenti sui fenomeni migratori, il gusto del rischio e dell'avventura di chi intraprende il viaggio. Il viaggio è al centro di gran parte della letteratura italiana sull'emigrazione del primo Novecento, soprattutto di quella divulgativa e popolare. Gran parte di questa produzione riprende gli stereotipi negativi del viaggio di emigrazione proposti dalla cultura dell'epoca (MARTELLI, 1994). La traversata viene presentata come la tappa di un esodo di cui sono protagonisti contadini poveri, ignoranti e straccioni. Una rappresentazione del viaggio non del tutto realistica, ma certo rassicurante per chi considera i flussi transoceanici un mezzo per allontanare dal paese ceti subalterni socialmente pericolosi (SANFILIPPO, 2015)

Negli anni della "grande migrazione" a bordo delle navi ci sono prevalentemente contadini, ma non mancano artigiani ed appartenenti ai settori del lavoro preindustriale che cercano opportunità di vita migliori inserendosi nel mercato internazionale del lavoro (CORTI, 2013). E', in genere, la miseria che spinge gli emigranti a varcare l'Oceano, ma questo non fa di loro dei soggetti assimilabili alle classi marginali e "pericolose" (CAMPESI, 2013). Gli emigranti sono spesso dei poveri, raramente dei miserabili. Chi vive in condizioni di miseria estrema e di marginalità sociale non solo non dispone di mezzi sufficienti per sostenere le spese del viaggio ma ha anche difficoltà a formulare un progetto migratorio. La rappresentazione dell'emigrante come soggetto sociale che grava sui destini del paese favorisce un'interpretazione in chiave di esodo dei flussi transoceanici. Il viaggio diventa un momento topico di separazione.

Il racconto letterario riprende ed esalta, nelle descrizioni della traversata, gli aspetti traumatici dell'esodo e contribuisce alla rappresentazione dei flussi transoceanici come un 
dramma sociale doloroso ma necessario. Di questo dramma la nave diventa il palcoscenico e il viaggio la trama. Nel racconto il viaggio assume valenze e significati che sono spesso lontani da quella che era l'esperienza dei migranti. Per molti di loro il viaggio non aveva particolari caratteristiche di eccezionalità, soprattutto perché poteva diventare una pratica quasi consuetudinaria. Come da tempo è stato documentato dalla storiografia (CORTI; SANFILIPPO, 2009), i flussi transoceanici prevedevano una mobilità tra due sponde molto elevata e non erano pochi gli emigranti che varcavano l'Oceano anche più volte in un anno. In questi casi, il viaggio non rappresentava certo un'esperienza "memorabile".

Esemplare della familiarità delle migrazioni storiche italiane con la traversata transoceanica, è la storia di un contadino ligure che è stato possibile ricostruire attraverso le tracce autobiografiche conservate nell'Archivio Ligure della Scrittura Popolare. ${ }^{2}$ Andrea Gagliardo, un piccolo proprietario terriero di una vallata del levante ligure, compi quattordici volte la traversata: la prima volta nel 1847, l'ultima nel 1888 (MOLINARI, 2005). Nella memoria autobiografica della sua esperienza migratoria, Gagliardo limita a pochi cenni telegrafici la descrizione: "1847. Brigantino Bettuglia da Genova a Nuova York. 57 giorni. 1861. Vapore Etna da Liverpool a New York. 17 giorni". ${ }^{3}$

Che la traversata sia percepita dagli emigranti non come un "luogo" ma come la "parentesi" di un cammino che ancora deve iniziare, è documentato dalla corrispondenza epistolare. Limitando l'osservazione alle più importanti raccolte edite di lettere, data la vastità di materiali di questo tipo disponibili in archivi pubblici e privati, si può osservare come sia molto limitato nella corrispondenza lo spazio che occupa la descrizione della traversata. Tra le più di cento lettere di contadini abruzzesi pubblicate nel 1913 da Filippo Lussana, Lettere di illetterati, (LUSSANA, 1913) solo in una compare la descrizione del viaggio transoceanico. Anche nel volume di Emilio Franzina, Merica! Merica! (FRANZINA, 1979) che raccoglie lettere di contadini veneti emigrati in Brasile nella seconda metà dell'Ottocento, i riferimenti alla traversata sono in genere limitati a poche e sintetiche annotazioni. E' solo quando sono particolarmente difficili che i viaggi compaiono nelle corrispondenze familiari. La descrizione della traversata spesso nelle lettere è poco più un incipit che precede la narrazione dell'arrivo a destinazione:

Dopo longo e borascoso mare trascorso, arivamo all'America, dove si credeva trovare le delizie della tera cioè lavorare poco e guadagnare molto, ma invece non è così, al contrario si lavora molto e si guadagna poco e si magna anche male perché i cibi non ano sustanza come quelli di Litalia (FRANZINA, 1979, p. 154).

Uno spazio maggiore, seppure sempre limitato rispetto al testo delle lettere, occupa il viaggio nella raccolta di epistolari di emigranti ticinesi in Australia curata da Giorgio Cheda (CHEDA, 1979). Le difficoltà che incontravano questi emigranti per arrivare nel paese di destinazione non erano certo paragonabili a quelle di chi emigrava in America. Già raggiungere il porto di imbarco poteva essere un viaggio lungo e faticoso. In una lettera del 1859, scritta subito dopo l'imbarco a Liverpool, un emigrante ripercorre giorno per giorno le tappe del 
viaggio per arrivare a Liverpool. Un viaggio che è durato circa venti giorni, in parte a piedi e poi utilizzando tutti i mezzi di trasporto disponibili: treno, carrozza, battello a vapore, slitta. Con tappe in varie città: Locarno, Bellinzona, Lucerna, Basilea, Parigi, Dieppe, Londra. Cosi descrive il suo passaggio delle Alpi:

9 gennaio: sono partito con la diligenza fino a Airolo. Da Airolo sull'alta montagna fino all'ospizio con la slitta a Goschenen e da qui a Fluelen su una carrozza. Da Fluelen a Lucerna su una carrozza a vapore (CHEDA, 1979, p. 50).

Sebbene meno lungo e avventuroso di quello degli emigranti ticinesi diretti in Australia, anche il viaggio degli emigranti italiani per raggiungere i porti di imbarco era spesso faticoso e pieno di ostacoli. C'erano poi le lunghe attese dell'imbarco in squallide locande o sulle banchine dei porti. I viaggi più faticosi erano però quelli a cui gli emigranti spesso dovevano sottoporsi, una volta sbarcati, per giungere a destinazione. Un viaggio che poteva durare anche un mese e che, soprattutto nel caso di chi era diretto in Argentina e in Brasile, avveniva spesso su mezzi di fortuna. Molti emigranti non si aspettavano, dopo aver attraversato l'Oceano, di dover continuare ancora il viaggio. Era un qualcosa "di troppo" rispetto ad un "cammino" già tanto lungo e faticoso. Non a caso è di questi viaggi, più che della traversata che si trova spesso traccia nella corrispondenza epistolare. In una delle lettere raccolte da Franzina un emigrante racconta con ricchezza di particolari il suo lungo viaggio, dopo lo sbarco in Brasile. Il viaggio transoceanico era stato di trenta giorni, quello per arrivare nella zona di colonizzazione ben più lungo e faticoso. Era durato quaranta giorni con i mezzi di trasporto più diversi e attraverso foreste e zone paludose. Alcuni emigranti che erano sopravvissuti alla traversata transoceanica, non riuscirono a sopportare le difficoltà di questo viaggio (FRANZINA, 1979, p. 90).

Nelle lettere degli emigranti la traversata transoceanica perde quelle caratteristiche di evento traumatico che invece assume nel racconto letterario. Anche quando compaiono resoconti dettagliati, il viaggio appare come una fatica, a volte come un'avventura pericolosa, raramente come un'esperienza drammatica di separazione. Chi attraversa l'Oceano sembra considerare l'evento migratorio come una separazione temporanea dal proprio paese più che come un "esodo" per terre lontane.

Solo quando, per circostanze diverse, il viaggio un "luogo" della memoria può venire "narrato" come una tappa di un percorso migratorio. Non a caso il viaggio diventa spesso un "racconto" nelle fasi finali dell'esperienza migratoria. Come documentano gli studi di Emilio Franzina, sono molte le autobiografie popolari, edite e inedite, in cui compare la narrazione del viaggio (FRANZINA, 1992; 2003). Questi di testi, pur differenziandosi dalle produzione letteraria sull'emigrazione, ne riflettono spesso gli stereotipi. C'è poi da osservare che si tratta di scritture di tipo autobiografico. Chi scrive la propria autobiografia, anche se è un emigrante appena alfabetizzato, lo fa per dare una "rappresentazione" di un percorso di vita che presume un lettore. Quello che Philippe Lejeune ha definito il "patto autobiografico" tra l'autore e il lettore (LEJEUNE, 1990). Questo tipo di produzione offre 
elementi importanti per documentare l'esperienza del viaggio transoceanico, ma conferma la natura del viaggio come soggetto letterario più che come oggetto di indagine storica.

L'interesse degli storici per gli aspetti soggettivi dell'esperienza migratoria ha fatto emergere dagli archivi familiari molti autobiografie inedite e ne ha favorito spesso la pubblicazione. Sebbene sia ormai molto vasta la produzione autobiografica disponibile per documentare il viaggio transoceanico, si tratta però un tipo di documentazione che mantiene un carattere di eccezionalità. Non sono pochi gli emigranti che hanno lasciato una traccia scritta della loro esperienza. Sono, però, un numero ben esiguo rispetto alla massa di coloro che per più di un secolo hanno varcato l'Oceano. E, questo, non solo per la difficoltà che hanno soggetti spesso appena alfabetizzati a praticare forme di scrittura più impegnative di una corrispondenza epistolare, soprattutto perché spesso manca agli emigranti lo stimolo a lasciare memoria di sé. La maggior parte di loro non ritiene di avere vissuto vicende "memorabili". Chi l'ha lasciato una memoria autobiografica è perché, o spontaneamente o per stimoli esterni, ha acquisito la consapevolezza che anche la vita della gente comune "merita" di essere raccontata.

Nel determinare la valenza del viaggio come "luogo" di memoria entrano in gioco fattori diversi. Certo la priorità delle motivazioni individuali, ma non sono certo ininfluenti le condizioni in cui il viaggio avviene. Se, ad esempio, ricorrenti sono nella autobiografie popolari del primo Novecento le descrizioni del viaggio come "Odissea" (STELLA, 2004) è perché restano fino ad allora poco sicure e certo non confortevoli le condizioni del viaggio. La frequenza dei naufragi, le pessime condizioni igieniche e sanitarie in cui spesso avveniva il viaggio, ne fanno una esperienza "memorabile". Anche se può esserci in queste narrazioni in eccesso di patos dovuto ad influenze più o meno dirette delle stereotipi letterari sul viaggio di emigrazione, resta il fatto che varcare l'Oceano presenta per più di mezzo secolo un'esperienza non priva di rischi per gli emigranti.

\section{Traffici e navi tra due sponde}

Il traffico di emigrazione si inserisce in una rete portuaria e di trasporti che è transnazionale già nel primo Ottocento. Ancora all'inizio del Novecento sono prevalentemente compagnie inglesi (Prince Line, Dominion Line, Cunard Line, Anchor Line, White Star Line) e tedesche (Hamburg America Line, Lloyd Bremen a fare scalo nei maggiori porti italiani) (TAYLOR, 1971; GLAZIER; DE ROSA, 1986; NUGENT, 1992). Il regime di monopolio in cui si trovò a operare l'unica grande compagnia italiana, la Navigazione Generale Italia, sorta, nel 1881, dalla fusione dei due gruppi armatoriali Florio e Rubattino, non rappresentò un serio ostacolo per gli interessi delle compagnie straniere. Ancora negli anni 1912-1913, quando i flussi migratori toccano le punte più alte, poco meno della metà del trasporto per mare degli emigranti viene svolto dalle compagnie tedesche e inglesi.

La storiografia, soprattutto quella italiana, ha dimostrato scarsa attenzione per le vicende del trasporto di emigrazione. Paradossalmente di un fenomeno di mobilità che riflette 
le dinamiche del mercato del lavoro internazionale è stato trascurata l'analisi del segmento che ne rappresenta la base di sviluppo: la rete dei trasporti e gli interessi ad essa collegati. Questo ha portato ad una sottovalutazione del ruolo svolto dal mercato dei trasporti marittimi nel determinare la direzione e la periodizzazione dei flussi. Una maggior attenzione alle vicende internazionali del trasporto di emigrazione avrebbe consentito, ad esempio, di verificare più precocemente i legami di continuità che vennero a stabilirsi tra l'emigrazione continentale e quella transoceanica, tra i primi flussi verso le Americhe e l'esodo di massa di fine Ottocento. L'imbarco dai porti europei è spesso il segnale di un cambiamento del progetto migratorio. Quando si verificano determinate condizioni (apertura di nuovi mercati del lavoro, fallimenti di progettualità migratorie individuali, attivazione di reti di richiamo dalle Americhe) le migrazioni stagionali di lavoro si trasformano in flussi migratori transoceanici (CORTI; ALBERA, 2000).

Nell'ambito della storiografia italiana le problematiche del trasporto di emigrazione sono state inserite nel contesto della storia dei ceti armatoriali e delle loro strategie imprenditoriali (DORIA, 1990). Il prevalere di un approccio di questo tipo ha lasciato poco spazio ad una valutazione degli effetti che lo sviluppo del trasporto transoceanico ebbe sulla storia sociale dell'emigrazione. Non è un caso che a tutt'oggi si sappia ancora poco su ciò che avveniva nei porti di imbarco e nel corso del viaggio.

Tra i porti italiani è quello di Genova a gestire per quasi un secolo la mole più consistente del traffico di emigrazione. Qui la quota degli imbarchi per le Americhe è già di notevoli dimensioni in epoca preunitaria. Questi flussi sono in parte da ascrivere alla precocità con cui si manifestano nell'area ligure i fenomeni migratori, anche se appare assai probabile che a imbarcarsi a Genova fossero anche emigranti provenienti da altre regioni. Nel periodo 1833-1850 sono circa 14.000, in base ai dati forniti dai registri dell'Ufficio di Sanità marittima del porto, gli emigranti che partono da Genova per le Americhe. Meta privilegiata di questi flussi erano le regioni del Plata (68\%), subito dopo venivano gli Stati Uniti (16,5\%) e il Brasile (8\%). Dal 1876 al 1901 nel porto di Genova si imbarca il 61\% dell'emigrazione transoceanica italiana. Negli anni successivi la meridionalizzazione dei flussi e la prevalenza delle correnti migratorie per gli Stati Uniti farà assumere al porto di Napoli il primato nel traffico di emigrazione. Già nel 1901 il porto di Napoli imbarca una quota di emigranti che il doppio di quella del porto di Genova. A partire dal 1905 prendono consistenza i traffici migratori nel porto di Palermo e con quote meno rilevanti anche nel porto di Messina. Il porto di Genova continuerà comunque a mantenere una quota consistente di imbarchi: il 34\% dal 1902 al 1924 (MOLINARI, 2001).

Il decrescere, nel primo Novecento, del traffico di emigrazione non influi sullo sviluppo delle attività marittime nel porto di Genova. Anzi, fu proprio negli anni di passaggio dall'Ottocento al Novecento che si verificò un particolare incremento dell'economia portuale sia nel traffico delle merci sia in quello dei passeggeri. Il ceto armatoriale genovese aveva infatti trovato nel trasporto di emigrazione una fonte di finanziamento che gli aveva consentito 
di consolidare, nell'ultimo ventennio dell'Ottocento, la sua posizione nell'ambito dei settori emergenti del capitalismo italiano: quello della siderurgia e quello della navalmeccanica (CASTRONOVO, 1990). Alcuni gruppi armatoriali genovesi avevano saputo sfruttare appieno le convergenze di interessi che erano venute a crearsi tra questi settori dell'industria e le aspirazioni espansioniste della sinistra storica. A beneficiare delle sovvenzioni statali alla marina mercantile, prima nel 1877 poi nel 1882, furono soprattutto i ceti armatoriali genovesi. I più conservatori nelle strategie imprenditoriali e i più accaniti difensori del trasporto a vela. Come ben documentano gli atti della Commissione d'inchiesta della marina mercantile (1881-1882), fu grazie alle concessioni fatte dal governo all'Associazione marittima ligure che gli armatori genovesi riuscirono ad inserirsi nel mercato del trasporto di emigrazione (TONIZZI, 2000). Le sovvenzioni assicurano all'economia marittima finanziamenti regolari e consistenti. Con la protezione dello stato le compagnie di navigazione genovesi acquistano una maggiore stabilità finanziaria e ad avviano una trasformazione degli assetti societari che vede la partecipazione di un capitale diversificato, industriale e finanziario.

La "modernizzazione" dei ceti armatoriali genovesi trova un elemento di forte incentivazione nell'assenza, fino al 1901, di una legge organica sull'emigrazione (SORI, 1979). Privo di un dispositivo di tutela, il trasporto degli emigranti diventa un investimento particolarmente vantaggioso per le compagnie. Ad una richiesta di imbarco continuamente in crescita si risponde adattando al traffico di emigrazione una flotta vecchia e desueta. Osserva a questo proposito, nel 1895, Giovanni Cantù, responsabile dei servizi sanitari del porto di Genova:

La marina italiana non è priva di buoni piroscafi. Il guaio è che della nostra emigrazione si fa un ignobile monopolio per trarre dal quale il maggior profitto possibile si adibisce pel trasporto materiale scadente, quasicchè gli emigranti fossero merce infima (CANTÙ, 1895).

Sulle rotte di emigrazione vengono utilizzate, ancora nei primi anni del Novecento, le "carrette del mare", vecchi piroscafi privi dei requisiti essenziali di sicurezza e di igiene. Non erano pochi gli emigranti che morivano durante il viaggio o che venivano respinti nei porti di arrivo perché gravemente ammalati. Il viaggio transoceanico era un'esperienza ad alto rischio. Si poteva fare naufragio, essere sbarcati in un paese diverso da quello di arrivo. La cosa che accadeva con maggior frequenza era di contrarre malattie contagiose a causa delle condizioni di affollamento e di sporcizia in cui avveniva la traversata (MOLINARI, 1988).

L'inserimento del ceti armatoriali e dell'economia marittima italiana nel contesto del traffico di emigrazione si rivela fin dall'inizio improntato a una strategia ben precisa: conciliare il massimo profitto con il minimo di investimenti. Non è certo un caso che lo sviluppo delle compagnie di navigazione genovesi, le prime a praticare il trasporto transoceanico degli emigranti, avvenga in concomitanza con le politiche di emigrazione gratuita e sovvenzionata promosse per buona parte dell'Ottocento dai paesi del Sud America (BRUNELLO, 1994; VANGELISTA, 1997). Ciò che consente al ceto armatoriale genovese di praticare con 
successo il traffico di emigrazione è la lentezza con cui avviene nella marina mercantile italiana il passaggio dalla vela al vapore. A differenza di altre forti marinerie a vela come, ad esempio, quella salernitana (FRASCANI, 2001), la marineria ligure già nei primi anni dell'Ottocento ha esteso le sue rotte dal Mediterraneo all'Atlantico. E' quindi in grado di far fronte alla crescente richiesta di trasporti transoceanici. Gli armatori liguri non hanno bisogno di investire grandi capitali per inserirsi nel traffico di emigrazione. E' sufficiente possedere un veliero di medie dimensioni per realizzare ingenti guadagni (DORIA, 1990).

In assenza un quadro legislativo di tutela e di controllo dei flussi migratori i ceti armatoriali genovesi operano spesso come "agenti" di emigrazione. Attraverso la rete dei loro intermediari fanno incetta di emigranti per i governi del Sud America e gestiscono il traffico dei biglietti "pre-pagati". Nel 1870 la società Lavarello si accorda, al di fuori di ogni canale diplomatico, con il presidente argentino Mitre per arruolare contadini da avviare alla colonizzazione. L'Argentina è un territorio privilegiato per le compagnie liguri. Qui esistono consolidati rapporti commerciali con la Liguria e una rete di relazioni politiche e diplomatiche che passano attraverso le Camere di commercio italiane, soprattutto quella di Buenos Aires (SCARZANELLA, 1983; BERTAGNA, 2009). Nel 1869 con il contributo degli immigrati liguri in Argentina viene costituita la "Società di navigazione italo-platense" che dispone di tre piroscafi per la linea Genova - Rio de Janeiro - Montevideo - Buenos Aires (MOLINARI, 2005).

I proventi che le società armatoriali genovesi traggono dal traffico di emigrazione favoriscono processi di concentrazione che portano alla formazione dei primi gruppi imprenditoriali e segnano la fine della figura dell'armatore-mercante. Nel 1871 nascono a Genova due grandi compagnie: il Lloyd italiano che, nel giro di pochi anni, dispone di una flotta di cinque grandi vapori e la Gio.Batta Lavarello e Compagnia, che all'epoca monopolizza gran parte del traffico di emigrazione per il Sud America. Ci sono poi altri importi gruppi armatoriali: quello Raffaele Rubattino, di Erasmo Piaggio, di Edilio Raggio (MOLINARI, 2001).

Sono i cambiamenti che investono il mercato internazionale del lavoro ad accelerare il declino dell'armatoria ligure. La forte richiesta di manodopera che viene dagli Stati Uniti, modifica la composizione, le dimensioni, le direttrici dei flussi transoceanici. Le nuove esigenze del trasporto marittimo impongono l'abbandono dalla navigazione a vela e la modernizzazione delle flotte. Delle centotrenta società armatoriali presenti a Genova nel 1873 poche riescono a sopravvivere al passaggio dal vecchio al nuovo secolo. Il decrescere dei flussi migratori per il Sud America da un lato; la difficoltà di adeguare le flotte agli standard del trasporto internazionale, dall'altro accentuano la fragilità economica dei gruppi armatoriali genovesi. Solo attraverso la fusione con la palermitana Società Florio il gruppo di armatori che fa capo alla Società Rubattino, riesce a mantenere un posto di rilievo nel traffico di emigrazione.

La formazione nel 1881 della Navigazione Generale Italiana, dove confluiscono i capitali delle società Florio e Rubattino apre la via a un processo di concentrazione degli interessi del settore che assumerà dimensioni sempre maggiori con il passare del tempo. 
Nel 1885 la N.G.I. acquisisce le flotte delle compagnie Raggio e Piaggio e si trova così a disporre dell'81\% della flotta a vapore del compartimento di Genova. Nel periodo 1890-1895 si assiste a Genova ad un potenziamento dell'apparato armatoriale che vede impegnata la finanza internazionale e i settori più avanzati del capitalismo industriale. I vecchi gruppi armatoriali liguri sono presenti con uomini e capitali nel gruppo della N.G.I, ma all'interno della compagnia svolgono ruoli sempre più marginali Sono gli anni in cui nel porto di Genova si registra un incremento del 50\% del traffico di emigrazione a cui si accompagna un'accresciuta domanda di trasporti per la prima guerra d'Africa. Decisivo è l'apporto del capitale tedesco che si riversa in questi anni in Italia, in particolare a Genova. La società La Veloce, fondata nell'1887, diventa nel 1889 di proprietà di alcune banche tedesche. Nel 1889 con capitali della Banca Commerciale viene costituita l'Italia società di navigazione a vapore per il trasporto di emigranti in Sud America. Nel 1898 aumenta la partecipazione tedesca alla N.G.I. e alla Veloce e viene stipulato un accordo tra queste due società e la Hamburg America Line per l'esercizio del traffico di emigrazione da Genova a Buenos Aires. Da parte sue la N.G.I continua a fagocitare le vecchie società sia genovesi che napoletane e siciliane e a incassare il 90\% delle sovvenzioni statali alla marina mercantile (MOLINARI, 2009).

Il traffico di emigrazione assume cosi negli anni di fine secolo la funzione di "volano" dell'economia nazionale perché in questo settore convergono gli interessi dei più grandi monopoli industriali e finanziari dell'epoca: la cantieristica e la siderurgia (CASTRONOVO, 1990). Come già era accaduto con il trasporto a vela e nelle prime fasi di passaggio al vapore, la favorevole condizione in cui si trova ad agire l'armatoria italiana, priva di fatto di vincoli legislativi nella gestione del trasporto degli emigranti e "protetta" dalla sovvenzioni statali, favorisce strategie imprenditoriali tese ad ottenere il massimo profitto con il minimo di investimenti. La stabilità dei noli, garantita dal flusso costante degli emigranti, si traduce in una rendita di posizione che ritarda il progresso tecnologico della flotta. Esemplare è a questo proposito la politica seguita dalla N.G.I. L'acquisto di un numero sempre crescente di pacchetti azionari delle altre società porta questo gruppo a mantenere sulle rotte di emigrazione una flotta che non è in grado di competere con quella delle compagnie straniere. Ancora nel 1897 l'età media delle navi della N.G.I addette al trasporto degli emigranti è di 23 anni. Un ufficiale della marina che ha viaggiato per molti anni sulle rotte dell'emigrazione, parla di queste navi come di "piroscafi - cimitero". Cosi le descrive in una pubblicazione del 1903:

Si vedono tuttora non poche vecchie, lente, instabili e insicure carcasse, senza alcuna garanzia d'igiene a bordo esercitare il traffico della nostra emigrazione per le Americhe, anzi qualcuna di queste vecchie navi ricorda i vascelli medievali stipati di schiavi (GABRIELLI, 1903, p. 15).

Solo alla vigilia della Grande Guerra, quando cominciano ad essere attivati i dispositivi di tutela previsti dalla legge del 1901 sul trasporto per mare degli emigranti (ispettorati dell'emigrazione nei porti, commissari viaggianti sulle navi) e viene previsto un pur blando dispositivo di controllo sullo stato del naviglio addetto al trasporto di emigrazione, si assiste 
al progressivo ammodernamento delle flotte. Sono però soprattutto le misure di controllo sulla "qualità" dei flussi migratori messe in atto dagli Stati Uniti ad accelerare, nel primo decennio del Novecento, il ritiro dalla rotte transoceaniche di una flotta vecchia e spesso priva di requisiti igienico-sanitari.

\title{
Le navi dei folli
}

Ancora nel primo ventennio del Novecento, le compagnie di navigazione, soprattutto quelle italiane, utilizzavano per il trasporto degli emigranti navi poco idonee alle rotte transoceaniche: vecchie, con scarsi requisiti di sicurezza, con limitata velocità (MOLINARI, 1988). Inoltre il numero degli emigranti imbarcati era in genere eccessivo rispetto agli spazi disponibili sulla nave. Le condizioni di affollamento e sporcizia dei dormitori cosi venivano descritte da un medico di "bordo":

\begin{abstract}
La temperatura non è il solo fattore che rende irrespirabile l'atmosfera dei dormitori, vi concorre il vapor acqueo e l'acido carbonico della respirazione, i prodotti tossic che svolgono dalla secrezione dei corpi, dagli indumenti dei bambini e talora degli adulti, che per tema o per pigrizia non esitano a emettere urine e feci negli angoli del locale dove stanno alloggiati. L'impressione di disgustosa ripugnanza che si riceve scendendo in una stiva dove hanno dormito gli emigranti e tale che, provata una sola volta non si dimentica più (CANTU், 1895, p. 13).
\end{abstract}

Che negli anni della Grande migrazione il viaggio transoceanico avvenisse in condizioni tali da mettere a rischio la sopravvivenza degli emigranti non rappresentava certo un problema per lo stato italiano. Basti pensare che, a proposito dei requisiti di igiene e sicurezza previsti le navi adibite al trasporto degli emigranti, legge del 1901 non presenta modifiche di rilievo rispetto al Codice della Marina Mercantile del 1879. Come se lo stato non si fosse reso conto delle dimensioni di massa assunte dai fenomeni migratori negli anni a cavallo tra Ottocento e Novecento. Sul ponte di coperta dei piroscafi la legge prevede uno spazio minimo di 0,45 metri cubi per ogni emigrante. E nei dormitori un boccaporto e una latrina ogni duecento posti letto (MOLINARI, 2009).

La burocratizzazione, a seguito della prima legge organica sull'emigrazione (1901) (OSTUNI, 2001) delle procedure di controllo sanitario degli emigranti sia all'imbarco che nel corso della traversata ha prodotto una mole imponente di "carte" da cui emerge un quadro a fosche tinte del viaggio transoceanico. Si tratta, prevalentemente, di giornali sanitari di bordo, di cartelle cliniche, di relazioni sanitarie inviate dai medici al Ministero dell'Interno e alla Direzione Generale della Sanità Pubblica. Una tipologia di fonti che, oltre a fornire un quadro delle condizioni igieniche e sanitarie del viaggio transoceanico, offre elementi utili per documentare i costi psico-fisici delle esperienze migratorie (MOLINARI, 2002). Non sono pochi gli emigranti che si imbarcano già ammalati, numerosi sono quelli che si ammalano nel corso delle traversate, molti quelli che tornano in patria perché gravemente ammalati. 
Sebbene la figura dell'emigrante abbia una valenza sociale di tipo biologico - in quanto pura forza-lavoro l'emigrante non può che essere un "corpo sano" (DALLAGO, 1999) - il tema della salute è stato raramente affrontato dagli storci dell'emigrazione. E' anche per questo che la documentazione sanitaria dei viaggi transoceanici appare di particolare interesse.

Sia nei viaggi di andata sia in quelli di ritorno, le navi assumono spesso il carattere di veri e propri "ospedali galleggianti". Nel viaggi di andata sono soprattutto le malattie contagiose ed epidemiche a mettere a rischio la salute degli emigranti. Scrive, nel dicembre 1906, un medico di bordo:

\begin{abstract}
Le condizioni sanitarie del viaggio di andata sono state poco soddisfacenti: sopra 1.401 emigranti ho avuto 48 ricoverati in infermeria di bordo dei quali solo tre guarirono durante la traversata, 44 sbarcarono ammalati ed uno ebbe esito letale. La causa di cosi grave percentuale di ammalati la si deve attribuire all'essersi sviluppate a bordo quasi contemporaneamente tre diverse malattie infettive: vaiolo, varicella e morbillo. ${ }^{4}$
\end{abstract}

Nel 1907, secondo i dati forniti raccolti dal Commissariato Generale dell'emigrazione, nei viaggi per il Sud America, su un totale di 47 morti, 24 erano bambini da 0 a 10 anni, 19 dei quali deceduti per morbillo; nei viaggi di ritorno vi erano stati 72 decessi, più della metà di bambini e lattanti, vittime di epidemie di morbillo (COMMISSARIATO GENERALE DELL'EMIGRAZIONE, 1926).

E' soprattutto nei viaggi di ritorno che la nave assume a pieno titolo il carattere di "ospedale galleggiante". Non sono pochi gli emigranti gravemente ammalati che tornano in patria per morire. Nei viaggi di ritorno dagli Stati Uniti sono la tubercolosi polmonare e le malattie mentali, a fornire gli indici statistici più elevati. In quelli di ritorno dal Sud America è particolarmente alto è il numero di affetti da tracoma. Notevole, sebbene meno elevata rispetto ai viaggi di ritorno dagli Stati Uniti, la percentuale di ammalati che presentano forme di alienazione mentale.

Nel periodo 1903-1925, sono 7.749, nei viaggi di ritorno dagli Stati Uniti, gli emigranti affetti da tubercolosi, più di duemila quelli classificati come malati mentali. Nello stesso periodo sono circa tremila gli emigranti che tornano dal Sud America ammalati di tracoma (MOLINARI, 2014).

Cosi, il medico di bordo del piroscafo Calabria descriveva, nel 1910, la situazione sanitaria dei migranti nel viaggio di ritorno da New York: "Tra i respinti vi erano dei tubercolotici, dei reumatici cronici, dei malarici. Nel viaggi di ritorno il piroscafo non era che una grande infermeria". ${ }^{5}$

La maggior rigidità dei controlli sanitari allo sbarco nei porti degli Stati Uniti, fa sì che qui, più che nei paesi del Sud America, molti emigranti siano "respinti" al paese d'origine perché considerati malati di mente. Dall'analisi dei giornali di bordo e dei giornali sanitari, si può rilevare come, tra il 1901 e il 1920, la diagnosi di mental insanity fosse la causa princi- 
pale del rimpatrio coatto o del respingimento allo sbarco degli immigrati italiani. Nel 1909, ad esempio, su un totale di 400 emigranti che tornano ammalati dagli Stati Uniti, 340 sono classificati come alienati mentali (MONTANO, 1910).

Dal 1902-1920, le alienazioni mentali sono al secondo posto, dopo la tubercolosi, nelle statistiche sanitarie redatte dal Commissariato dell'emigrazione (MOLINARI, 2010). Gran parte degli emigranti che ritornano in patria come alienati mentali sono stati "respinti".

Negli Stati Uniti gli emigranti che non avevano la possibilità di provvedere alle spese mediche e si rivolgevano a strutture assistenziali e sanitarie, correvano il rischio di essere rimpatriati. La legislazione sull'immigrazione conteneva norme che prevedevano il divieto di ingresso nel paese a soggetti "mentalmente disturbati". Nel 1882 viene approvato il primo decreto federale che proibisce lo sbarco degli alienati e dei minorati mentali. Per i trasgressori si stabilisce il rimpatrio forzato. Sempre in base a questa norma il migrante che, a distanza di un anno dall'arrivo negli Stati Uniti, ricorreva a strutture di assistenza psichiatrica, era "respinto" al paese di origine. Un decreto sull'immigrazione, del 1903, proibisce lo sbarco agli epilettici e a coloro che abbiano avuto esperienze di ospedalizzazione per malattie mentali nei cinque anni precedenti. Nel 1907 questo decreto è integrato con altre norme restrittive. Viene impedito l'ingresso negli Stati Uniti a chi, al momento dello sbarco, si comporti in modo da apparire inadatto al lavoro. Un decreto del 1917 specifica quali sono le malattie mentali che precludono l'ingresso nel paese. Sono considerati da "respingere" gli psicopatici, gli alcoolisti, i frenastenici, gli "agitati" (CAMPESI, 2013).

Dei migranti "respinti" o rimpatriati per malattia mentale solo una parte, al rientro in Italia, veniva ricoverata in manicomio. A partire dal 1904, con la prima legge organica sul funzionamento dei manicomi, sono previste procedure di assistenza e di controllo sugli emigranti rimpatriati con diagnosi di malattia mentale. Si trattava, però, di normative difficili da attuare a causa della mancanza nei porti italiani di strutture di assistenza agli emigranti (MOLINARI, 2002). Dalle storie cliniche pubblicate su alcune riviste di psichiatria si può rilevare come il ricovero in manicomio non avvenisse, in genere, dopo lo sbarco, ma dopo periodi più o meno lunghi dal rientro dell'emigrante al paese di origine.

Lo spoglio delle maggiori riviste di psichiatria e di neuro psichiatria degli anni a cavallo tra Ottocento e Novecento evidenzia la scarsa attenzioni degli ambienti medico-psichiatrici per il la diffusione di patologie mentali tra gli emigranti.

Non è la sofferenza psichica degli emigranti a preoccupare la medicina dell'epoca, quanto, piuttosto, il timore per il rientro di persone considerate socialmente pericolose. Significativi appaiono, a questo proposito, alcuni articoli pubblicati sulla "Rivista di emigrazione". 6 In uno di questi, Antonio D'Ormea, direttore del manicomio di Pesaro, evidenzia la difficoltà di attuare misure di controllo sul rientro dagli Stati Uniti di migranti "respinti" perché malati di mente: "I medici dei porti di sbarco a cui mi sono rivolto per avere notizie degli emigranti alienati nemmeno seppero darmi il numero di questi infermi. I manicomi cui essi fanno capo al loro sbarco sono troppi e non tengono conto della speciale distinzione 
degli emigranti" (D'ORMEA, 1909, p. 6).

Nei viaggi di ritorno dagli Stati Uniti, i medici di bordo si limitavano a confermare la diagnosi di mental inasanity che compariva nel provvedimento di espulsione. Accadeva così che controlli volti a stabilire lo stato di efficienza fisica della manodopera immigrata, si trasformassero in una classificazione medica che faceva degli emigranti dei malati mentali. Bastava un malore per la stanchezza del viaggio, uno stato di confusione provocato dalle burocratiche procedure di controllo nei porti di sbarco, perché un emigrante venisse classificato come folle e "respinto". Una pratica che rientrava nella routine dei viaggi di emigrazione. Come confermano le sintetiche annotazioni dei medici di bordo sugli emigranti "respinti". Si legge in una relazione sanitaria del 1908:

Dei 100 italiani respinti nel porto di New York come alienati, 10 erano già alienati in patria, 45 avevano dato segni di irritazione durante il viaggio, 4 erano ubriachi, 30 erano sembrati bizzarri ai medici perché gesticolavano, 5 perché avevano dichiarato di avere parenti lontani ricoverati in manicomio.?

Non si dispone di informazioni attendibili per documentare cosa accadesse agli emigranti che ritornavano in Italia con problemi di salute mentale. E' probabile che chi riusciva a ricongiungersi alla famiglia, si sottraesse, almeno temporaneamente, al ricovero in manicomio. Diversa la situazione per chi non poteva contare su reti familiari. In questo caso i medici di porto si rivolgevano alle autorità di Pubblica Sicurezza che provvedevano al ricovero degli emigranti in manicomio.

Un approccio diverso da quello dominate nella psichiatria del tempo emerge da due ricerche sul rapporto tra migrazioni e malattia mentale pubblicate su "Il Manicomio moderno", la rivista del manicomio di Nocera Inferiore (SALOMONE, 2004). Di una è autore di Francesco Lener, Le malattie mentali e le correnti migratorie nell'Italia meridionale (1908 e 1909); dell'altra, Emigrazione e pazzia (1908) Giuseppe Tolone, un medico del manicomio di Girifalco (Cosenza) (LENER; 1908; LENER, 1909; TOLONE, 1908). In entrambi questi lavori, sebbene in modo più marcato in quello di $\mathrm{F}$. Lener, la malattia mentale è considerata un effetto dello sfruttamento economico e dell'isolamento sociale dei migranti sia in patria che all'estero. Lener prende in esame i migranti ricoverati a Nocera Inferiore dal 1905 al 1907, in totale 87 e pubblica le loro storie cliniche. Si tratta di maschi, provenienti dagli Stati Uniti, prevalentemente contadini, compresi in una fascia di età che va dai diciannove ai cinquantacinque. La maggior parte ha svolto lavori dequalificati e faticosi: muratori, minatori, sterratori, facchini. I ricoverati presi in esame rappresentano il 53\% degli ammessi nel manicomio nel triennio. Un dato di per sé indicativo, come rileva Lener, del ruolo svolto delle esperienze migratorie nel favorire il manifestarsi della malattia mentale. Le diagnosi che compaiono con maggior frequenza sono quelle di frenosi alcolica e di demenza precoce. Osserva Lener, nel valutare le cause predisponenti la malattia mentale: "Sono tre i fattori che danno il contributo maggiore ai fenomeni mentali morbosi tra gli emigranti: l'alcolismo, la sifilide, il lavoro" (LENER, 1908, p. 210). 
Dalle sintetiche storie cliniche presentate da Lener emerge un'uniformità di esperienze e "destini" migratori: lavori dequalificati e pesanti, isolamento sociale, solitudine da alleviare con le pratiche del bere. I ricoverati sono uomini che hanno affrontato da soli l'esperienza migratoria. Molti hanno svolto lavori manuali nelle zone del nord-est degli Stati Uniti, New Jersey e Pennsylvania. Su 87 emigranti ricoverati, solo tre sembrano aver trovato opportunità occupazionali che hanno consentito loro di accumulare qualche piccolo capitale. Quasi per tutti è segnalato l'abuso di bevande alcoliche che, per un numero consistente (20), si accompagna al contagio sifilitico. Esemplare la storia di I.D., un contadino che emigra all'età di diciassette anni:

Stette nell'America del Nord per cinque anni. Fece abuso di alcool e si contagiò di sifilide. Lavorò nelle miniere. Venne ricoverato in manicomio dopo cinque giorni dal rimpatrio. Eredità negativa (LENER, 1908, p. 231).

Per quanto sintetiche, queste storie lasciano intravvedere vite di isolamento e di solitudine. Questi emigranti non sembravano aver trovato nel paese di destinazione reti di parenti o di compaesani. E, forse, per la difficoltà di realizzare un progetto migratorio, hanno mantenuto scarsi rapporti con familiari e parenti rimasti in patria. Solo per due di loro, viene riferito che sono stati fatti rimpatriare dai parenti. Nella maggior parte dei casi il ricovero in manicomio avviene non molto tempo dopo il rimpatrio. Chi tornava a casa senza risorse economiche e con disturbi mentali, diventava un "peso" per la famiglia. L'esperienza migratoria sancisce una rottura di legami e di reti sociali che solo il successo di un progetto migratorio può, in parte, attenuare.

Non sempre era facile individuare gli emigranti tra i ricoverati in manicomio. Il che impediva di cogliere il rapporto tra sviluppo delle malattia e le esperienze migratorie. Un problema messo in rilievo da Giuseppe Tolone, in una ricerca sui migranti ricoverati nel manicomio di Girifalco (Cosenza) negli anni 1903-1906:

Suppongo con fondatezza che il numero degli emigranti ricoverati sia maggiore di quello da me rilevato. Ma disgraziatamente spesso si conosce poco o nulla dei ricoverati. Molti vengono condotti in manicomio da persone che poco li conoscono e nulla si ricava dai monchi e grami certificati che li accompagnano (TOLONE, 1908, p. 34).

Tolone fornisce informazioni sulle vicende migratorie dei ricoverati: "I 56 casi sono tutti di reduci dall'America del Nord, come risulta dalla parte storica anamnestica delle singole tabelle nosologiche. Alcuni impazzirono durante il viaggio di andata e furono quindi retrocessi in patria; altri subito dopo giunti alla meta, sicchè furono ricoverati in manicomi esteri e poi rimpatriati" (TOLONE, 1908, p. 45). Come già aveva rilevato Lener per il manicomio di Nocera Inferiore, anche in quello di Girifalco i migranti rappresentano una quota consistente dei ricoverati nei primi anni del Novecento: il 24\% nel periodo 1903-1906. I ricoverati sono tutti uomini di età compresa tra i diciannove e i quarantacinque anni. A differenza degli migranti ricoverati a Nocera Inferiore, quasi tutti contadini, quelli di Girificalco sono 
prevalentemente artigiani: ciabattini, falegnami, sarti. Alto è il numero dei migranti che sono stati respinti allo sbarco o hanno subito forme di rimpatrio coatto.

Le storie cliniche raccolte da Tolone restituiscono frammenti di un'umanità particolarmente povera e dolente. Il trauma intrinseco all'esperienza migratoria, sia come rottura di legami affettivi e sociali sia come incertezza del proprio destino, appare con particolare efficacia in alcune storie cliniche. Tra queste, quella di un giovane che, nel 1903, parte per andare negli Stati Uniti:

Venanzio C. 19 anni, celibe, calzolaio. Si recava a Baltimora in cerca
di lavoro, ma durante il viaggio per il dubbio di essere respinto da
quelle autorità per mancanza di una persona che lo accompagnasse,
diè tali segni di alienazione mentale che gli fu impedito lo sbarco
e venne perciò rimpatriato. Dalla famiglia, recatasi a Napoli ad
incontrarlo, fu condotto subito qui. Sempre triste e solitario.
Diagnosi: frenosi depressiva. Eredità negativa (TOLONE, 1908, p. 34).

Sono molti i casi in cui il fallimento di un progetto migratorio al momento dello sbarco provoca una reazione mentale che diventa una patologia. Dei 53 emigranti presi in esame da Tolone, molti sono stati "respinti" allo sbarco. Alcune storie cliniche documentano il trauma psichico di chi vedeva fallire un progetto migratorio già all'arrivo. E' il caso di quella di un sarto, sposato e padre di tre figli che emigra, nel 1905, negli Stati Uniti:

Nel novembre scorso Domenico G. si recò a New York, ma colà giunto gli fu vietato lo sbarco non si sa per quale ragione. Tornato in patria cominciò dapprima col mostrarsi svogliato al lavoro, in seguito non volle uscire di casa, mangiava poco. Viene perciò ricoverato in manicomio. L'infermo è accolto in uno stato depressivo estremo. E' abulico, inerte, si straccia spesso le vesti. Diagnosi: stato melanconico. Eredità negativa (TOLONE, 1908, p. 41).

Giuseppe Tolone, come Francesco Lener, considera la malattia mentale degli emigranti una conseguenza di traumi economici e sociali. Solo chi trova "reti" di sostegno nei paesi di destinazione può sopravvivere senza cadere nella miseria e nella follia: "I più forti sono quelli che incontrano situazioni che li rendono forti. Sono quelli più fortunati" (TOLONE, 1908, p. 37).

Dei traumi fisici e psichici vissuti da masse di emigranti negli anni di maggior sviluppo dei flussi transoceanici, sono rimaste poche tracce. A parte qualche sintetica e frettolosa certificazione della malattia riportata sui giornali sanitari di bordo e su riviste mediche del primo Novecento. Allora come oggi, il migrante è soprattutto un "corpo" che, quando si ammala, diventa un "peso", sia nella società di destinazione sia in quella di origine. Il manicomio ha svolto, storicamente, la funzione di "deposito" di persone considerate corpi socialmente inutili (BASAGLIA, 1968). Nel caso degli emigranti, come documentano gli articoli pubblicati su "Il Manicomio moderno", la funzione di segregazione e di alienazione della persona svolta dall'internamento manicomiale assume un carattere particolarmente punitivo. L'emigrante, a differenza di altri ricoverati, quando entra in manicomio si lascia alle spalle due vite: quella che ha trovato e quella che, invano, ha cercato altrove. 
Le dimensioni che assumono nei paesi di destinazione la tubercolosi, il tracoma, le malattie mentali non possono che suscitare interrogativi sul carattere di patologia sociale di massa di queste malattie. Anche se allo stato attuale degli studi non è possibile documentare l'esistenza di un nesso diretto tra la il manifestarsi della malattia e il contesto di vita e di lavoro degli emigranti, non sembra però difficile supporre che questo nesso esista. Come documentano i giornali sanitari di bordo e alcune cartelle cliniche, gran parte di chi ritornava sulle "navi ospedale" aveva alle spalle un percorso migratorio di sfruttamento e di emarginazione. Gli emigranti che si ammalavano nei paesi di destinazione avevano difficoltà a disporre di assistenza e di cure.

Che, nei viaggi di ritorno, le navi fossero spesso simili a "ospedali galleggianti" è il cinismo implicito nella "registrazione" sanitaria della sofferenza a documentarlo. Sintetica, ma efficace, a questo proposito, la conclusione della relazioni sanitaria di un viaggio di ritorno dagli Stati Uniti:

La salute dei rimpatriati lasciò molto a desiderare: molti cachetici, anemici, vere nullità fisiologiche. I rimpatriati dal Nord America o sono ammalati di affezioni croniche, specie all'apparato respiratorio e cardiovascolare, o sono convalescenti cagionevoli, invalidi, molti i neurastinici. Insomma pochi i buoni (MOLINARI, 1988, p. 173).

\section{Referências}

AUGÉ, M. Non luoghi. Introduzione ad un'antropologia della surmodernità. Milano: Feltrinelli, 1993.

BASAGLIA, F. L'Istituzione negata. Torino: Einaudi, 1968.

BERTAGNA, F. La stampa italiana in Argentina. Roma: Dozelli, 2009.

BLENGINO, V. Oltre l'Oceano. Gli immigrati italiani in Argentina. Roma: Edizioni Associate, 1990.

BRUNELLO, P. Pionieri. Gli italiani in Brasile e il mito della frontiera. Roma: Donzelli, 1994.

CAMPANINI, M. La traversata. Racconto e rappresentazione del viaggio di emigrazione oltreoceano. Storie, memorie, voci. Lucca: Fondazione Paolo Cresci, 2010.

CAMPESI, G. La detenzione amministrativa degli stranieri. Storia, diritto, politica. Roma: Carocci, 2013. 
CANTÙ, V. L'igiene a bordo dei piroscafi addetti al trasporto degli emigranti. Rivista di igiene e di sanità pubblica, n. 17, 1895.

CASTRONOVO, V. L'industria italiana dall'Ottocento a oggi. Milano: Mondadori, 1990.

CHEDA, G. L'emigrazione ticinese in Australia. Locarno: Armando Editore, 1979.

COMMISSARIATO GENERALE DELL'EMIGRAZIONE. Annuario statistico dell'emigrazione italiana 1901-1926. Roma: 1926

CORTI, P; ALBERA, D. (ed). La montagna mediterranea una fabbrica di uomini? Mobilità e migrazioni in una prospettiva comparata. Cuneo: Gribaudo, 2000.

CORTI, P; SANFILIPPO, M. (ed). Migrazioni. Storia d'Italia. Annali. Torino: Einaudi, 2009.

CORTI, P. Temi e problemi di storia delle migrazioni italiane. Viterbo: Sette Città, 2013.

D'ORMEA, A. Per la profilassi psichica dei nostri emigranti. Rivista di Emigrazione, n. 2, p. 1-7, 1909.

DALLAGO, A. Non-persone. L'esclusione dei migranti in una società globale. Milano: Feltrinelli, 1999.

DORIA, G. Debiti e Navi. Genova: Marietti, 1990.

FRANZINA, E. L'immaginario degli emigranti. Miti e raffigurazioni dell'esperienza italiana all'estero tra due secoli. Treviso: Pagus, 1992.

FRANZINA, E. Merica! Merica! Emigrazione e colonizzazione nelle lettere dei contadini veneti in America Latina (1876-1902). Milano: Feltrinelli, 1979.

FRANZINA, E. Traversate. Le grandi migrazioni transatlantiche e I racconti italiani del viaggio per mare. Foligno: Editoriale Umbra, 2003.

FRASCANI, P. (ed.) A vela e a vapore. Economie, culture e istituzioni del mare nell'Italia dell'Ottocento. Roma: Donzelli, 2001.

GABRIELLI, P. La nostra marina mercantile. Napoli: Tipografia Gennaro Errico e figli, 1903.

GLAZIER, I.; DE ROSA, L. Migration across Time and Nations: Population Mobility in Histor- 
ical Context. New York: Holmes \& Meyer, 1986.

L'EMIGRAZIONE italiana (1870-1970). Roma: Pubblicazioni degli archivi di stato, 2002.

LEJEUNE, P. H. /l patto autobiografico. Bologna: Il Mulino, 1990.

LENER, F. Le malattie mentali e le correnti migratorie nell'Italia meridionale. Il Manicomio Moderno, n. 20, p. 183-248, 1908.

LENER, F. Le malattie mentali e le correnti migratorie nell'Italia meridionale. Il Manicomio Moderno, n. 21, p. 177-186, 1909.

LUSSANA, F. Lettere di illetterati. Note di psicologia sociale. Bologna: Zanichelli, 1913.

MARTELLI, S. Letteratura contaminata. Storia, parole, immagini tra Ottocento e Movecento. Salerno: Laveglia, 1994.

MOCHI, G (ed.), R. L. Stevenson. Emigranti per diletto seguito da Attraverso le pianure. Torino: Einaudi, 1987.

MOLINARI, A. Emigrazione e follia nel primo Novecento. Rivista sperimentale di Freniatria, n. 3, v. 84, p. 47-65, 2010.

MOLINARI, A. La salute degli emigranti. In: BEVILACQUA, P.; DE CLEMENTI, A.; FRANZINA, E. (ed.). Storia dell'emigrazione italiana. Arrivi. Roma: Donzelli, 2002. vol. II, p. 377-395.

MOLINARI, A. Le navi di Lazzaro. Aspetti sanitari dell'emigrazione transoceanica italiana: il viaggio per mare. Milano: FrancoAngeli, 1988.

MOLINARI, A. Les migrations italiennes au début du XXe Siécle. Le voyage transocéanique entra évenemént et récit. Torino-Paris: L'Harmattan, 2014.

MOLINARI, A. Porti, trasporti, compagnie. In: BEVILACQUA, P.; DE CLEMENTI, A.; FRANZINA, E. (ed.). Storia dell'emigrazione italiana. Partenze. Roma: Donzelli, 2001. v. 1, p. 237-256.

MOLINARI, A. Traversare. In: CORTI, P.; SANFILIPPO, M. (ed.). Migrazioni. Storia d'Italia. Annali. Torino: Einaudi, 2009. p. 529-548.

MOLINARI, A. Traversate. Vita e viaggi dell'emigrazione transoceanica italiana. Milano: Ed. Selene, 2005. 
MONTANO, A. Statistica degli emigranti ricoverati nelle infermerie di bordo nell'anno 1909. Bollettino dell'emigrazione, n. 26, p. 11-30, 1910.

MORICOLA, G. (ed.). Il viaggio degli emigranti in America Latina tra Ottocento e Novecento. Napoli: Guida Ed., 2000.

NUGENT, W. Crossing: The Great Transatlantic Migrations (1870-1914). Bloomington: Indiana University Press, 1992.

OSTUNI, M. R. Leggi e politiche di governo nell'Italia liberale e fascista. In: BEVILACQUA, P.; DE CLEMENTI, A.; FRANZINA, E. Storia dell'emigrazione italiana. Partenze. Roma: Donzelli, 2001. vol. I, p. 309-321.

SALOMONE, G. Il manicomio di Nocera Inferiore. Napoli: Idelson - Gnocchi, 2004.

SANFILIPPO, M. (ed.). Migrazioni. Storia d'Italia. Annali. Torino: Einaidi, 2009.

SANFILIPPO, M. Nuovi problemi di Storia delle migrazioni italiane. Viterbo: Sette Città. 2015.

SCARSANELLA, E. Italiani d'Argentina. Venezia: Marsilio, 1983.

SORI, E. L'emigrazione italiana dall'Unità alla seconda guerra mondiale. Bologna: Il Mulino, 1979.

STELLA, G. Odissee. Italiani sulle rotte del sogno e del dolore. Milano: Rizzoli, 2004.

TAYLOR, P. H. The distant Magnet. European Emigration to the U.S.A. New York: Haper, 1971.

TOLONE, G. Emigrazione e pazzia. II Manicomio Moderno, n. 20, p. 29-66, 1908.

TONIZZI, M. E. Merci, strutture e lavoro nel porto di Genova tra '800 e '900. Milano: Franco Angeli, 2000.

VANGELISTA, C. Dal vecchio al nuovo continente. L'immigrazione in America Latina. Torino: Paravia, 1997.

\section{Notas}

1 Per un quadro della documentazione sull'emigrazione transoceanica italiana negli archivi pubblici e privati: Ministero per i beni e le attività culturali (L'EMIGRAZIONE, 2002).

2 Per la storia dell'Archivio e per un catalogo dei materiali di scrittura popolare: www.alsp.unige.it

3 Copia microfilmata del diario/memoria di Andrea Gagliardo è conservata nell'Archivio ligure della scrittura 
popolare dell'Università di Genova.

4 Archivio centrale dello stato (d'ora in poi ACS); Ministero dell'Interno; Direzione Generale di Sanità pubblica. Relazioni sanitarie (1882-1915), p. 992.

5 ACS (Archivio Centrale dello Stato), DGSP ( Direzione Generale di Sanità Pubblica), (1882 - 1915), p. 95, Relazioni sanitarie. Relazione sanitaria del piroscafo Calabria.

6 La "Rivista di Emigrazione" nasce nel 1908 ed è sostenuta, anche finanziariamente, dagli ambienti economici più filoemigrazionisti, Viene stampata a Pesaro. Tra i collaboratori: P. Ascoli, direttore della Scuola Superiore di Commercio, A. Alberti, direttore del manicomio di Pesaro, A. Cabrini, responsabile dell'ufficio emigrazione della Società Umanitaria, i medici e accademici A Mosso e P. Cogliolo, N. Colajanni, U. Conti, responsabile dell'Istituto di studi coloniali, l'economista G. Mortara.

7 ACS, DGSP, b. 52, Relazioni sanitarie (1901 - 1912), Relazione sanitaria del piroscafo "Città di Torino".

Augusta Molinari. Docente Titulare di Storia Contemporanea - Facoltà di Scienze della Formazione dell'Università di Genova. Corso Andrea Podestà, 2, 16121 - Genova, Itália.

Recebido em 27/10/2016

Aprovado em 07/11/2016 